\title{
Enhancements to the Planar Active Handrest
}

\author{
Mark A. Fehlberg, Hamidreza N. Sani, and William R. \\ Provancher \\ University of Utah
}

The Planar Active Handrest (PAHR) is a large workspace assistive device that improves user precision manipulation. The PAHR is restricted in its use, because it does not rotate in the horizontal plane, which limits the workspace size and reduces user comfort. This paper evaluates an improved device, the Enhanced Planar Active Handrest (E-PAHR), which allows lateral/medial rotation of the upper arm. Under the E-PAHR design, the desired hand position can be obtained with redundant arm and device configurations. As such, we gave consideration to controller input choices and resultant device motions that are available. Three experiments evaluated the controller designs to select the most effective method to control our device. We conclude that a rotational DOF (degrees of freedom) allows the E-PAHR to better follow the kinematics of a user's planar arm movements while allowing skill level equal to the PAHR, with reduced user force input and lower perceived exertion.

Keywords: human-robot interaction, man-machine systems, assistive devices, adaptive control, motion analysis

\section{Introduction}

The previous version of the Planar Active Handrest was a Cartesian two-degrees-of-freedom (DOFs) human-robot cooperative interface that provided support while performing dexterous tasks over large workspaces. The PAHR provided user support from a 2-axis computer-controlled motorized $x-y$ stage that allowed planar movements with a fixed forearm orientation. Previous studies with this device demonstrated that use of the PAHR provides increased task accuracy and user skill level compared to freehand, fixed, and passive repositionable supports (Fehlberg, Gleeson, \& Provancher, 2012). Additional studies showed that user skill level could be further improved by adding virtual fixtures to the device's controller (Fehlberg, Nisky, Doxon, \& Provancher, 2014). In the research presented herein, we endeavor to improve the PAHR device to better match human arm kinematics by adding a rotational DOF and to investigate its effect when interacting with this device.

Humans tend to have difficulty performing precision tasks without additional physical support. To alleviate this problem, static supports have been used to steady a human's arm or hand motions in performing activities of daily life. For instance, humans traditionally use the surface of a table to support their wrist while writing, which has been shown to reduce muscle activity and

\footnotetext{
Authors retain copyright and grant the Journal of Human-Robot Interaction right of first publication with the work simultaneously licensed under a Creative Commons Attribution License that allows others to share the work with an acknowledgement of the work's authorship and initial publication in this journal.
}

Journal of Human-Robot Interaction, Vol. 4, No. 3, 2015, Pages 149-169, DOI 10.5898/JHRI.4.3.Fehlberg 
may reduce fatigue (Ito \& Yokokohji, 2009). However, the inability to reposition stationary support and being limited to the hand's workspace is a major drawback of static supports. Traditional tools used to solve this issue, such as a maulstick used by painters, require the user to reposition the device, which does not fully solve the problem.

For our work described here, we developed an Enhanced Planar Active Handrest (E-PAHR) to provide additional ergonomic/kinematic support over a larger planar workspace (see Fig. 1). This device has three DOFs: two in translation and one in rotation. Through our investigations of this new device, we will enhance the workspace of the current PAHR to better match the kinematics of the human arm by developing a variety of methods to control the E-PAHR's redundant DOFs.

This paper presents three experiments evaluating the utility of E-PAHR controller methods and their effect on human movement. The first experiment examines preliminary controller methods to down-select the most effective input/controller strategies based on participant performance and subjective feedback. The second experiment examines improvements/modifications to the three most effective strategies from the first experiment. The third experiment compares E-PAHR's controller strategies to the original PAHR design and the Freehand drawing condition to evaluate the trade-off between speed and accuracy and the effectiveness of each condition, where effectiveness is expressed in terms of total applied force, efficiency, and perceived exertion of test participants.

The following section provides a brief background concerning the literature relevant to our research. The background section is followed by a description of the Enhanced Planar Active Handrest and the control strategies we investigated. Next, we outline the general methods used in all our experiments. Each of the three experiments is then presented in turn, with individual methods and results subsections. Finally, results from all experiments are summarized and future work is discussed.

\section{Background}

Various methods of hand and arm support have been used to increase precision and reduce fatigue while performing dexterous tasks. A simple example of a fixed static support is using a table to

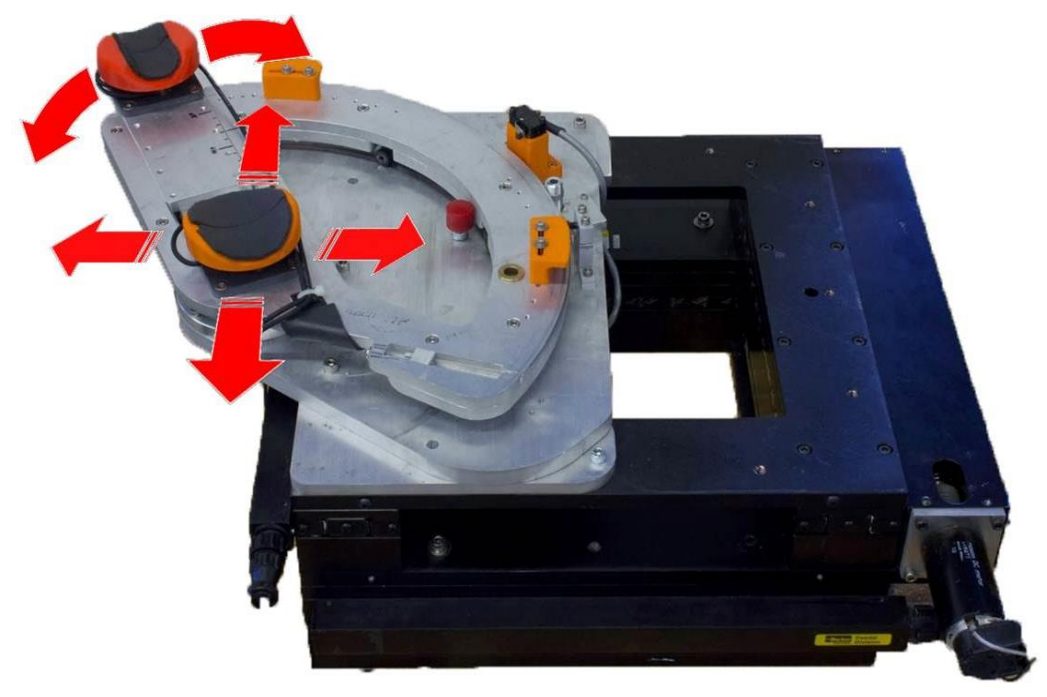

Figure 1. Enhanced Planar Active Handrest Prototype with arrows depicting device degrees-of-freedom/motions. 
stabilize the wrist while writing. Repositionable static supports reduce muscle fatigue by reducing the weight of the arm while typing (Erdelyi, Sihvonen, Helin, \& Hanninen, 1998) and using tools (Equipois, 2015). Passive (Chyatte, Long $2^{\text {nd }}$, \& Vignos Jr., 1965; Yasuda, Bowman, \& Hsu, 1986) and active (Beer, Naujokas, Bachrach, \& Mayhew, 2008) mechanical supports are also designed to reduce muscle fatigue through gravity compensation of the user's arm.

Robotic arm support devices have been used for both rehabilitation and to assist healthy users in performing dexterous tasks. In rehabilitation, robotic devices are often used in conjunction with virtual environments to motivate patients to achieve a higher intensity rehabilitation, which has been shown to improve rehabilitation of stroke patients (Kwakkel, Wagenaar, Koelman, Lankhorst, \& Koetsier, 1997). Robot-assisted repetitive task-oriented motion has been used as an effective means of upper arm rehabilitation (Riener, Nef, \& Colombo, 2005). Other benefits of such rehabilitation devices are improving grip strength throughout the first year after stroke (Langhammer, Lindmark, \& Stanghelle, 2007) and increasing elbow extensions in the horizontal plane using active gravity support of the arm (Beer, Ellis, Holubar, \& Dewald, 2007).

Robotic devices have also been used to assist healthy users to perform dexterous tasks with higher skill level. The Steady Hand, a robotic system developed at Johns Hopkins University, helps users with sub-millimeter manipulation tasks by simultaneously taking force input from the user and a robot to move the tool accordingly (Taylor et al., 1999). A "Cobot" is a human robot cooperative device that assists the user by constraining motion to a desired trajectory (Colgate, Wannasuphoprasit, \& Peshkin 1996).

Common designs of robotic arm supports include endpoint manipulation and powered exoskeletons. Endpoint manipulation rehabilitation devices guide a user's hand along a predefined trajectory to regain lost motor skills in their arm (Hogan, Krebs, Sharon, \& Charnnarong, 1995; Loureiro, Amirabdollahian, Topping, Driessen, \& Harwin, 2003; Lum \& Burgar, 2002). In contrast, exoskeletons are typically anthropomorphic robots that are tightly connected to the user's arm and its joint angles fully define the arm's pose (Moubarak, Pham, Moreau, \& Redarce, 2010; Nef, Guidali, \& Reiner, 2009). The E-PAHR is a hybrid of these designs where our focus is to provide highly accurate endpoint motions without the constraints found in typical exoskeletons.

Another consideration in the design of robotic arm supports is the controller choice, which is often coupled to the device's design. The two major categories of robotic controllers are impedance and accommodation control. Impedance control is used on lightweight, highly backdrivable devices that exert a force on the user based on the device's position (Hogan, 1985).

Accommodation control is used on heavier, non-back-drivable devices. This control technique allows the device to move in a particular direction with an applied virtual damping relative to an input force (Mathewson \& Newman, 1995; Matsuo \& Iwaki, 1992; Whitney, 1977). Admittance control adds a virtual mass in addition to the virtual damping of an accommodation controller. Accommodation and admittance controllers have been used in many robotic applications, such as commercial haptic interfaces (Van der Linde, Lammertse, Frederiksen, \& Ruiter, 2002), rehabilitation robotics (Colombo et al., 2005), and eye surgery with the Johns Hopkins' Steady Hand robot (Taylor et al., 1999). In both controllers a high accommodation/admittance gain (i.e., low damping) results in high device velocity, which leads to poor user precision. A low accommodation/admittance gain can be used to achieve the highest level of precision. However, a low accommodation/admittance gain also causes a reduction in the device velocity and can result in user fatigue when the user exerts higher forces than necessary (Wise \& Shadmehr, 2002). This suggests that there is an ideal range of accommodation/admittance gains and device velocities for optimal user accuracy and that our device's controller must be designed and tuned appropriately to achieve optimal user performance with low fatigue.

The speed and accuracy trade-off in human movements has been studied extensively. The trade-off was first described by Woodworth (1899) and later mathematically formalized as Fitts' law (Fitts, 1954). Fitts' law has been observed in a wide variety of tasks and applications (see Plamondon \& Alimi, 1997 for a review). The majority of the research on trade-offs between speed 
and accuracy is related to reaching movements. However, recent analysis has focused on more complicated movement sequences (Reis et al., 2009) and tracing tasks (Shmuelof, Krakauer, \& Mazzoni, 2012). This analysis suggested that the speed-accuracy trade-off function relates to a change in skill level (Krakauer \& Mazzoni, 2011; Reis et al., 2009; Shmuelof, Krakauer, \& Mazzoni, 2012). In our studies, we assess the effect of different device controller conditions on the skillfulness of user movements during tracing tasks.

\section{Device Description and Control Strategies}

In this section, we describe the development of the E-PAHR and the control strategies we examined. The development of the E-PAHR (Fig. 2) extends the workspace of the currently existing planar solution PAHR to better match the kinematics of the human arm. The design of the E-PAHR adds a third, rotational DOF to allow lateral and medial arm rotations in the horizontal plane. The E-PAHR is based on a Parker two-axis linear stage with an available translational workspace of $25 \times 25 \mathrm{~cm}$, with software and hardware limits of $20 \times 20 \mathrm{~cm}$ for safety of the device. On top of the Parker stage we have added a custom rotary stage that uses a capstan drive system with a gear ratio of 34:1. Two ATI Mini40 6-axis force-torque sensors measure user force interactions with our device. The elbow force sensor is placed directly on the center of the rotation axis beneath a padded support. The hand force sensor is placed beneath a second padded support at the end of an adjustable support arm whose length is adjusted to accommodate each user's forearm length between 15 and $30 \mathrm{~cm}$. The 2 Mini40 force sensors are calibrated to a $+/-20 \mathrm{~N}$ full scale range in the horizontal direction with a resolution of $0.005 \mathrm{~N}$.

While interacting with the E-PAHR, the user's right hand and/or wrist is rested on the forward padded support. The user's right elbow rests on the rear padded elbow support. For our tracing tasks, the user also grasps the stylus of a SensAble Technologies Phantom Premium 1.5 with his or her right hand.

The Active Handrest's position (X, Y, and Theta) is measured by three 1000-count-perrevolution optical encoders operating in quadrature. Position data are collected by a Sensoray 626 data acquisition card at a frequency of $2 \mathrm{kHz}$. Force input data are collected by 2 National Instruments PCIE-6320 DAQ cards. Position information from the Phantom Premium's stylus is

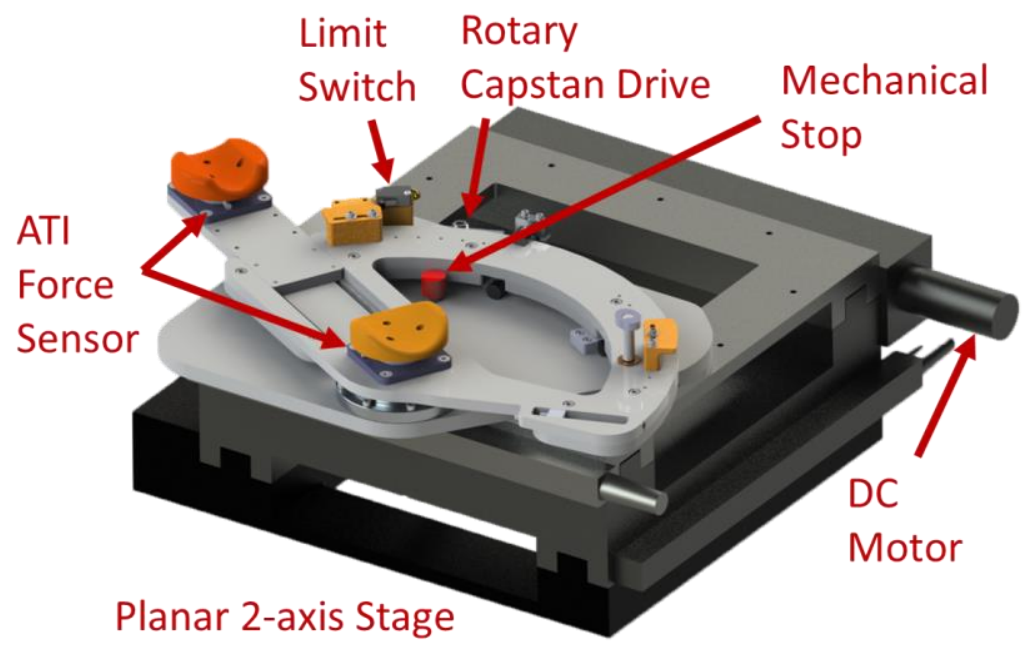

Figure 2. Enhanced Planar Active Handrest (E-PAHR) Schematic. 
Fehlberg et al., Enhancements to the Planar Active Handrest

processed using Phantom drivers, version 4.2 and OpenHaptics Academic Edition version 3.0. The input data are processed by our controller on a $2.66 \mathrm{GHz}$ Intel Core2-Quad PC operating in a 32bit Windows 7 environment, running $\mathrm{C}++$ code with CHAI-3D libraries. The computed motor commands are sent through the Sensoray 626 card and amplified by Advanced Motion Controls 12A8 PWM current amplifiers before being sent to the $x-y$ stage's 2 Maxon RE40 and the rotary axis's Maxon RE36 DC motors.

We chose to use a capstan drive for the rotational axis to increase the torque of the motor and reduce its speed similar to a gear but without any backlash that a user could haptically feel. Using such a system allows us to increase the reachable workspace without increasing the overall size of the device, increase robustness to singularities, and increase dexterity (Ueberle, Mock, \& Buss, 2004). However, adding a third DOF to control a two-DOF human arm, assuming that the shoulder is fixed (a widely used assumption in upper limb motion tracking literature (Goncalves, Di Bernardo, Ursella, \& Perona, 1995; Moeslund \& Granum, 2000), results in an over-defined system.

\section{E-PAHR Controller Strategies}

The controllers used in this paper consist of five accommodation controller variations and one admittance controller. The controllers compute the two desired translational motor velocities that are computed by using the control law:

$$
V=K_{a} * F_{\text {input }}
$$

where $\quad V$ - velocity

$K_{a}$ - accommodation/admittance gain ( inverse of damping)

$F_{\text {input }}$ - force input

For all controller strategies, $K_{a}$ was generally set at $0.010(\mathrm{~m} / \mathrm{s}) / \mathrm{N}$ based on previous device performance as measured in Fehlberg, Gleeson, \& Provancher, 2012 and Fehlberg et al., 2014). We then implemented a closed-loop PID controller to achieve this desired translational velocity. The desired rotational velocity, $\omega$, is also computed by the control law modified for rotations as:

$$
\omega=K_{r} * T_{\text {input }}
$$

where $\quad K_{r}$ - rotational accommodation/admittance gain ( inverse of damping)

$T_{\text {input }}$ - torque calculated from the interaction forces at the user's contact points with the device as measured by the two force/torque sensors and not the torque measured directly by the force/torque sensors.

For all controller strategies, $K_{r}$ was set as $K_{a} /(\text { forearm length })^{2}(\mathrm{rad} / \mathrm{s}) / \mathrm{Nm}$. The dependency of this gain on the forearm length allows the rotational and translation damping to be equivalent (e.g., a force applied by the hand only causes the same motion as an equivalent torque input). In this case, we first integrated the rotational velocity to achieve a desired rotation position and then implemented a PD controller to achieve this desired rotation position. The PD controller's gains were empirically tuned during pilot studies $\left(\mathrm{K}_{\mathrm{P}}=70\right.$ and $\left.\mathrm{K}_{\mathrm{D}}=0.02\right)$. We did not directly control the velocity of the rotational axis due to the back-drivable nature of the capstan drive mechanism.

As shown in (Nambi, Provancher, \& Abbott, 2011), human force control ability and precision deteriorates at low forces. However, this deterioration can be avoided by using a deadband to allow the stage to stop at force levels below a certain threshold. Therefore, to increase the stability of the E-PAHR, we added dead-bands to the translational and rotational motions such that no motion would occur with input forces less than $0.5 \mathrm{~N}$. 
Fehlberg et al., Enhancements to the Planar Active Handrest

Table 1. Controller and support conditions examined.

\begin{tabular}{|c|c|c|c|}
\hline $\begin{array}{l}\text { Controller } \\
\text { Strategy/Support } \\
\text { Condition } \\
\end{array}$ & Support & $\begin{array}{l}\text { Controller } \\
\text { Type }\end{array}$ & Description \\
\hline Basic & E-PAHR & Accommodation & $\begin{array}{l}\text { Sum of applied hand and elbow forces mapped to stage } \\
\text { translations. Moment from hand and elbow forces mapped } \\
\text { to device rotation }\end{array}$ \\
\hline Force Following & E-PAHR & Accommodation & $\begin{array}{l}\text { Applied hand forces mapped to stage translations. Elbow } \\
\text { moves in the direction of elbow forces while attempting } \\
\text { to have no effect on desired hand motion }\end{array}$ \\
\hline $\mathrm{R}-\mathrm{R}$ & E-PAHR & Accommodation & $\begin{array}{l}\text { Applied hand force controls stage "rotary joint" motions } \\
\text { and rotation axis }\end{array}$ \\
\hline $\mathrm{R}-\mathrm{R}+$ Prismatic & E-PAHR & Accommodation & $\begin{array}{l}\text { Applied hand forces control the device's "rotary joint" } \\
\text { motions and rotation axis; elbow moves radially in the } \\
\text { direction of the elbow force against a virtual damper }\end{array}$ \\
\hline $\begin{array}{l}\text { Instantaneous Center } \\
\text { of Rotation (ICR) }\end{array}$ & E-PAHR & Accommodation & $\begin{array}{l}\text { Forearm rotational velocity is calculated about a point } \\
\text { where the hand and elbow force vector normals intersect }\end{array}$ \\
\hline $\begin{array}{l}\text { Integrated Free Body } \\
\text { Acceleration (FBA) }\end{array}$ & E-PAHR & Admittance & $\begin{array}{l}\text { Applied hand and elbow forces determine free body } \\
\text { translational and rotational accelerations, which are then } \\
\text { integrated to determine stage translations and rotations }\end{array}$ \\
\hline No Rotation & PAHR & Accommodation & $\begin{array}{l}\text { Non-rotating stage. Applied hand forces mapped to stage } \\
\text { translations }\end{array}$ \\
\hline Freehand & None & N/A & Unsupported \\
\hline
\end{tabular}

To determine how best to control the E-PAHR, we examined a variety of control strategies in order to obtain a controller that provides the user with the most natural interaction and accuracy with the device. The five accommodation control and one admittance control strategies we examined are highlighted in Table 1. The first control strategy, "Basic," uses the average of the two force sensors to determine the device's translational velocity. The torque used to determine the rotational velocity is calculated from the elbow and hand input force components perpendicular to the forearm's axis. This control strategy was examined as it was computationally the simplest.

The second control strategy, "Force Following," uses the technique often employed by exoskeletons. In this strategy, the hand force sensor determines the position of the device's end effector (i.e., the user's hand). The force input at the elbow force sensor causes stage motion of the user's elbow in the direction of applied force in an attempt to minimize any force generated by the elbow, while not effecting the hand's position. This controller strategy was believed to be of value, because it would allow for precise end effector control.

The third controller strategy, "R-R," uses a planar human arm model with a fixed shoulder position to essentially transform the two prismatic joints of the stage into a second rotary joint (see Fig. 3 for schematic). The advantage of this control strategy is that the elbow force sensor is not required. The forth strategy, "R-R + Prismatic," is similar to the third strategy but it allows for additional elbow repositioning by allowing elbow movement radially inward and outward (i.e., along $l_{1}$, as shown in Fig. 3). In this configuration, movement of the first rotary joint is no longer fixed to an arc. To achieve this additional motion, only a single axis of the elbow force sensor is required. Both of these controller strategies could lead to a less expensive device, as the elbow force sensor is either not required or can be replaced by a single-axis force sensor. 


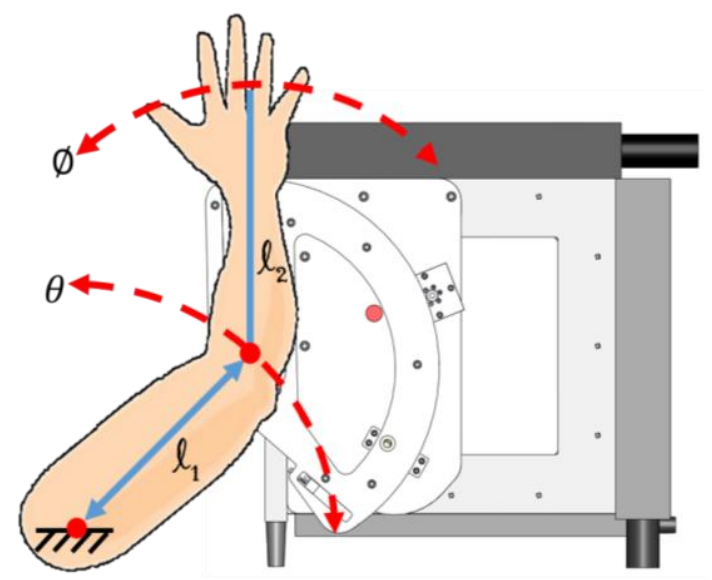

Figure 3. Rotary-Rotary (R-R) and Rotary-Rotary-Prismatic (R-R + Prismatic) controller concepts. $\ell 1$ and $\ell 2$ are fixed lengths for the $R-$ $\mathrm{R}$ controller. $\mathrm{l} 1$ is variable for the $\mathrm{R}-\mathrm{R}+$ Prismatic controller.

The fifth control strategy calculates the Instantaneous Center of Rotation, or "ICR," of the stage. For this strategy, the forearm is treated as a rigid body with tensile/compressive forces along the forearm recorded for efficiency analysis but not used in the controller calculations. The ICR location is calculated as the intersection of the vector normal of the elbow and hand force inputs. The forearm body is then rotated about this ICR. The E-PAHR's motion is calculated to achieve this motion of the forearm relative to the ICR. This strategy was examined as it allowed for the use of a single accommodation gain (i.e., no difference between rotation and translational damping).

The final E-PAHR strategy, and only classical admittance controller, is the integrated Free Body Acceleration model, or "FBA" In this strategy, we treat the forearm as two equal-point masses connected by a massless rigid bar. To keep the admittance controller comparable to the accommodation controllers without a virtual mass, $0.3 \mathrm{~kg}$ was selected as the smallest mass that still allowed for controller stability. We calculate the translational acceleration by solving for $a$, from:

$$
m * a=F_{\text {input }}-c * V
$$

where $\quad m$-sum of the two point masses

$F_{\text {input }}$ - average of the two force inputs

$c$ - damping (inverse of the admittance gain, $K_{a}$ )

$V$ - actual translational velocity

The velocity of the device is then calculated by integrating the calculated acceleration. Similarly, the rotational acceleration is calculated by solving for $\alpha$ in:

$$
I * \alpha=T_{\text {input }}-c * \omega
$$

where $I$ - mass moment of inertia of the two point masses

$T_{\text {input }}$ - torque measured across the two force inputs

$c$-damping (inverse of the rotational admittance gain, $K_{r}$ )

$\omega-$ actual rotational velocity 
The rotational velocity of the device is then calculated by integrating the calculated rotational acceleration. This controller strategy had the benefit of not only being able to specify different rotational and translational admittance gains but different translational and rotational inertias if desired.

The non-rotating PAHR and Freehand conditions will be used as references to compare our current strategies to the results obtained with various E-PAHR controller strategies.

\section{General Methods}

For the purpose of our device evaluation, we examined participants' performance while tracing 2D shapes over the course of 3 different experiments. We first compared performance and subjective preferences with the various E-PAHR controller conditions. Based on this analysis, we selected the three best controller methods and then attempted to improve them by making controller modifications. Finally, we compared the best modified E-PAHR configurations to performance under the PAHR and Freehand (unsupported) conditions based on performance, subjective feedback, and participant effort/exertion measures.

To evaluate the performance of these strategies, we used similar tracing tasks as used to evaluate the PAHR in Fehlberg et al. (2014). We had participants explore shapes with various spatial features (e.g., open vs. closed/cyclic paths and paths with continuously changing curvature vs. paths with discontinuous changes in curvature). Differences have been found between rhythmic (smooth) and discrete (segmented) movements (Ben-Tov, Levy-Tzedek, \& Karniel, 2012; Hogan \& Sternad, 2007; Levy-Tzedek, Ben-Tov, \& Karniel, 2011; Schaal, Sternad, Osu, \& Kawato, 2004). Using a variety of path shapes (see Table 2) allows us to assess the performance of the E-PAHR in both smooth and segmented movements.

\section{Experimental Apparatus}

For each experiment, the participant sat in front of the monitor and grasped the Phantom's stylus with his/her right hand, as shown in Fig. 4. For each of the E-PAHR and PAHR conditions, the participant placed his/her right elbow on the rear elbow support and his/her right wrist/hand on the

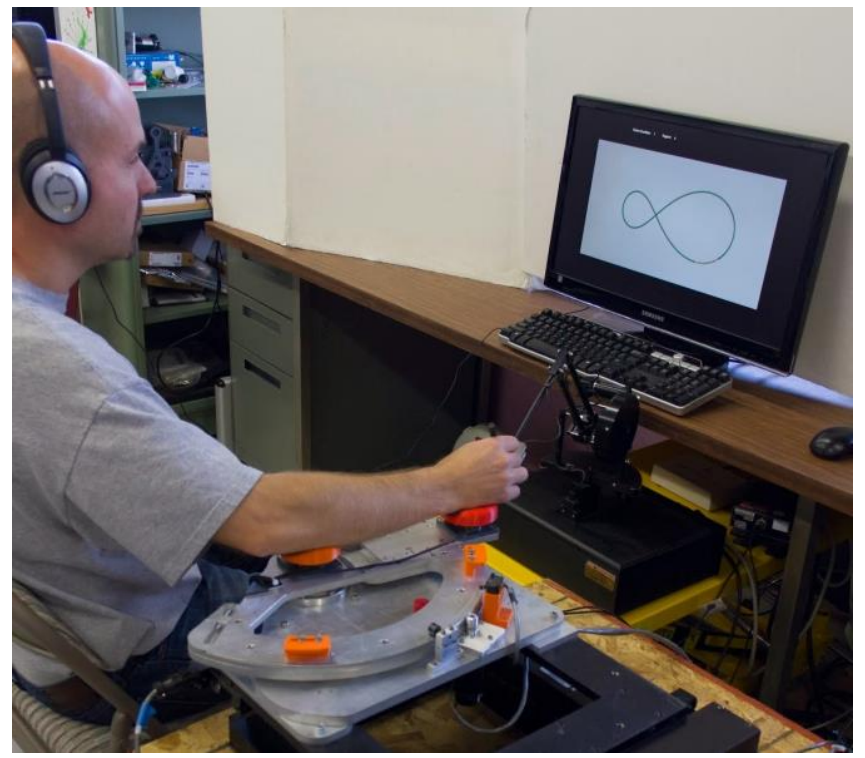

Figure 4. Experiment setup with target shape displayed on screen. 
Fehlberg et al., Enhancements to the Planar Active Handrest

forward hand support. For the PAHR condition, the participant rested only his/her elbow as they would normally, but the rotational axis was held in a fixed orientation. In the Freehand condition, the participant moved in an unsupported manner and did not rest or support his/her right arm. We did not consider either a Fixed Hand or Fixed Elbow support because the size of the shapes being traced by participants often exceeded the limited reach of a fixed support. A Passive support (i.e., a repositionable gravitational support without damping) was also not considered in this experiment, as we previously determined this mode of support to be less effective than the Freehand (unsupported) condition (Fehlberg et al., 2012).

\section{Experimental Procedures}

Before beginning each experiment, the length of the forearm support (i.e., the distance between the elbow, hand supports, and force sensors) was adjusted for the participant's comfort. Our controller then automatically adjusted the rotational accommodation/admittance gain to accommodate for the longer (or shorter) moment arms. Before starting each controller condition we asked the participant to navigate the perimeter of the workspace to allow the participant to feel comfortable with each condition and to ensure the Phantom and E-PAHR were able to reach the entire workspace. The E-PAHR and Phantom were repositioned as required for each participant's needs. Participants wore noise-cancelling headphones playing white noise throughout the experiments to reduce distractions.

One of the 5 virtual environment shapes was presented on the screen as a $2 \mathrm{~mm}$ wide black channel with a green centerline. The participants were instructed to "trace the target line as quickly and accurately as possible." Each shape was originally displayed on a red background with a green start block located on an edge for closed shapes or at an end for open shapes. The virtual environment background would change from red to grey when the participant first touched the green start block, indicating that experimental data was being recorded. A yellow trace line would be displayed indicating the traced path as the participant navigated the environment. The participant would navigate to the red stop block in order to complete each trial. After completing the trial, the participant would press the 'space bar' to advance to the next trial.

Table 2. Virtual Environment Shapes and Their Descriptions.

Description/Properties
$\begin{aligned} & \text { Closed, continuous, changing curvature without a change in direction } \\ & \text { of curvature } \\ & \text { Lemniscate }\end{aligned}$
Closed, continuous, changing curvature with a change in direction of
curvature


Data Analysis

Experimental data were collected at $2 \mathrm{kHz}$ from when the participant first touched the start block until the participant touched the stop block. The following information was measured for each trial:

- Median Error - median distance from the center-line

- Completion Time - amount of time to complete the trial

- Completion Time Per Distance - amount of time to complete the trial normalized per meter of path length (measured in $\mathrm{sec} / \mathrm{m}$ )

- Total Integrated Force - hand and elbow force inputs summed and integrated over time, as a measure of effort to complete each drawing task

- Total Integrated Force per Distance - Total Integrated Force normalized per meter of path length (measured in $\mathrm{N} \mathrm{sec} / \mathrm{m}$ )

- Total Effective Integrated Force - dot product of force input vectors with the associated hand and elbow velocity vectors

- Efficiency - Total Effective Integrated Force divided by Total Integrated Force times 100

- Subjective data***

- How "natural" each condition felt

- Participant's perception of accuracy

- Participant's perception of exertion

*** Due to the low sampling of subjective data (one sample per participant per condition), no statistical information other than the mean is presented for each category.

Participants were asked to rate each controller condition on a Likert scale (Likert, 1932) for the statements, "this condition felt natural to use" and "this condition allowed me trace the target accurately." Questions were answered using the following 5-point scale:

1. Strongly disagree

2. Disagree

3. Neither agree nor disagree

4. Agree

5. Strongly agree

Table 3. Borg's RPE Scale

\begin{tabular}{cl}
\hline Rating & Description \\
\hline 6 & No exertion at all \\
7 & Extremely light \\
8 & Very light \\
9 & \\
10 & Light \\
11 & Somewhat hard \\
12 & \\
13 & Hard \\
14 & Very hard \\
15 & \\
16 & Extremely hard \\
17 & Maximum exertion \\
18 & \\
19 & \\
20 &
\end{tabular}


When asked to rate their perceived level of exertion, participants responded using the Borg's Rating of Perceived Exertion (RPE) scale (Borg, 1982). They responded using the 6 to 20 point revised RPE (Borg, 1990) as shown in Table 3. For a reference, participants were told that " 9 very light" was "equivalent to walking slowly at your own pace," "13 - somewhat hard" was "equivalent to exercise they could sustain at that level of exertion," and "17 - very hard" was "equivalent to exercise where they would eventually fatigue."

For each experiment, we implemented a balanced Latin Squares scheme to control for condition carry-over effects, such as learning and fatigue. This scheme presents the experiment conditions in a different order for each participant. We conducted factorial ANOVA on each experiment's resultant data to determine significance of the manipulated variables. Statistical significance was determined at the 0.05 threshold level. All interaction terms were found to be non-significant unless specifically stated otherwise. All non-orthogonal post hoc contrasts were evaluated using Tukey's HSD to prevent against family-wise accumulation of Type-I error in multiple comparisons. All participants signed an informed consent form as stipulated by the University of Utah Institutional Review Board before participating in the experiments. All participants self-reported that they were right-hand dominant for all experiments.

\section{Experiment 1 - Preliminary Controller Evaluations}

The purpose of our first experiment was to determine the effectiveness of the various E-PAHR controller conditions. In this experiment, we compared participants' performance while tracing sine waves, oblate limacons, asymmetric lemniscates, and rectangles.

\section{Preliminary Controller Methods}

Oblate limacons, asymmetric lemniscates, sine waves, and rectangles were presented for each of the controller conditions examined. The experiment was completed by 6 participants ( 5 males and 1 female, ranging in age from 22 to 33 years). The time required to complete the experiment was approximately 30 minutes. The main factor of interest in this experiment was the controller condition. Each participant completed eight trials (two repetitions each of oblate limacons, asymmetric lemniscates, sine waves, and rectangles), under each of six E-PAHR controller conditions shown previously in Table 1 .

We first used a two-way $8 \times 4$ factorial ANOVA to predict median error and mean completion time per path length by controller condition and shape type with participant number entered as a random variable. We then used a two-way $8 \times 4$ factorial ANOVA to predict total integrated force and efficiency by controller condition and shape type with participant number entered as a random variable. Finally, we examined the mean participant preferences for their ratings of how natural each controller felt and how well they thought they performed with each condition.

Preliminary Controller Results and Discussion

Controller condition did not significantly predict median error $(F[5,75]=1.29, p=.30)$ (Fig. $5(\mathrm{a}))$. Shape Type did not significantly predict median error $(F[3,75]=1.20, p=.34)$. Before conducting completion time analyses, we normalized the completion time per path length. Controller condition also did not significantly predict completion time per path length $(F[5,75]=$ $2.10, p=.10]$ (Fig. 5(b)). Shape type, however, did significantly predict completion time per path length $(F[3,75]=4.27, p=.02]$. Rectangles took approximately $15.3 \mathrm{~s} / \mathrm{m}$ less time to complete than asymmetric lemniscates and sine waves.

Controller condition significantly predicted total integrated force per path length, which is an indicator of the effort required during each trial $(\mathrm{F}[5,75]=4.95, \mathrm{p}<.01)$ (Fig. 5(c)). The Basic and FBA controllers had lower total integrated force per path length than the R-R and R-R + Prismatic controllers $(\mathrm{p}<0.010)$. Shape type did not significantly predicted total integrated force per path length $(\mathrm{F}[3,75]=1.88, \mathrm{p}=.14)$. Controller condition also significantly predicted 
Fehlberg et al., Enhancements to the Planar Active Handrest
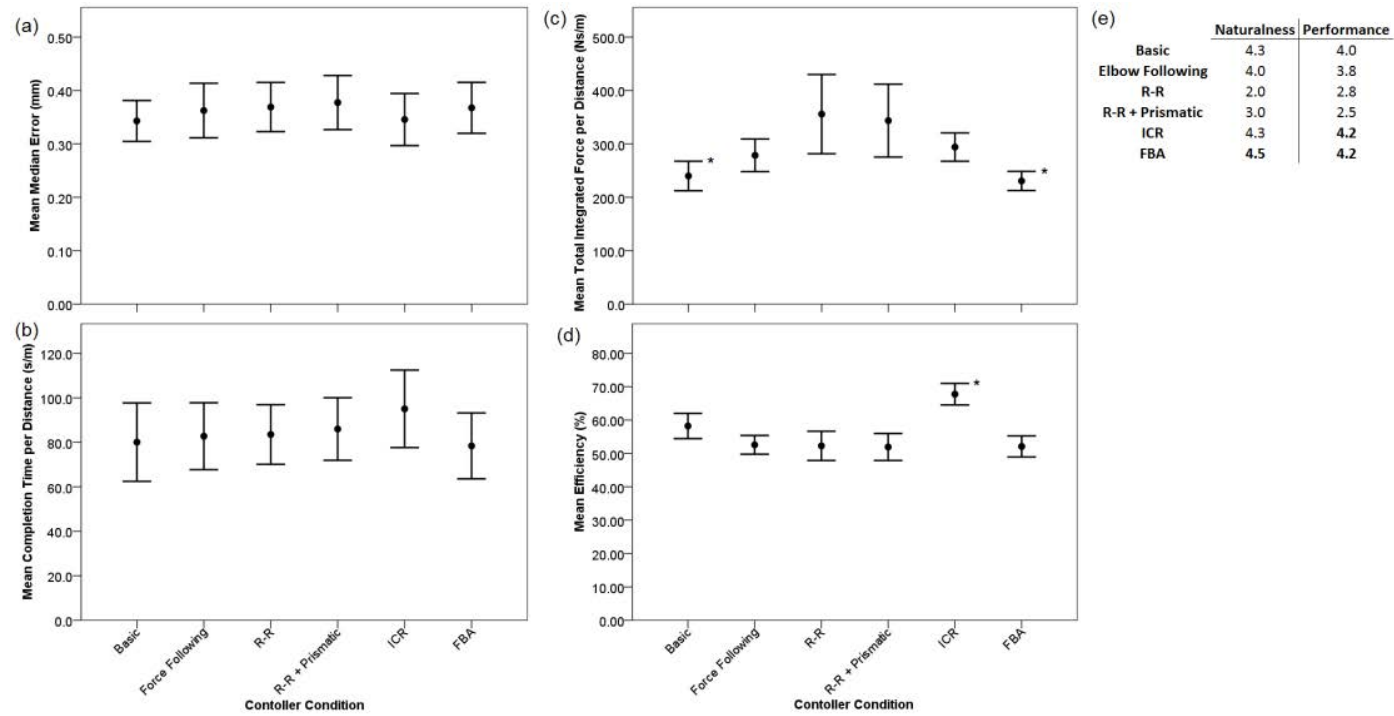

Figure 5. Experiment 1 Median Error (a), Completion Time per Distance (b), Total Integrated Force per Distance (c), Efficiency (d), and Subjective Ratings (e) by Contoller Condition. (Error bars indicate $95 \%$ confidence intervals for the estimated mean.)

efficiency $(\mathrm{F}[5,75]=13.05, \mathrm{p}<.01)$ (Fig. 5(d)). The ICR controller had higher efficiency than any of the other controller conditions $(\mathrm{p}<.01)$. Shape Type did not significantly predict efficiency $(\mathrm{F}[3,75]=.14, \mathrm{p}=.94]$.

The objective data from this experiment indicated that the Basic and FBA controllers required the least amount of total user effort (in the form of integrated force input) and that the ICR controller was the most efficient controller (had the highest force input efficiency). We also looked to the subjective data to confirm the most preferred conditions (Fig. 5(e)). When asked how natural the controller condition felt, participants most preferred the FBA condition (4.5/5.0), followed by the ICR and Basic conditions (4.3/5.0 for both). When asked how well they perceived that the controllers performed, participants felt that they did the best with the FBA and ICR conditions (4.2/5.0), followed by the Basic conditions (4.0/5.0). Based on these findings, we decided to investigate the Basic, ICR, and FBA conditions in our subsequent experiments. We would also like to point out that objectively, any of the other three controller conditions could achieve the same accuracy and timing data. Additionally, the two R-R conditions could be used with our device with fewer force-sensing inputs, but these also had the greatest total user effort required as seen by the integrated force and were the least subjectively preferred conditions.

\section{Experiment 2 - Controller Enhancement Comparison}

To further refine our choices for the controller options of the E-PAHR, we next made modifications to the three selected controllers from Experiment 1. User comments from our preliminary controller experiments described that the E-PAHR was often more difficult to move in rotation than in translation. We believe this is because we performed torque computations for our Basic and FBA controllers based on locating the center of mass at the midpoint between the elbow and hand. In our second experiment, we chose instead to perform torque computations based on locating the center of mass at a point located at one-quarter of the user's forearm length measured from the elbow position. The longer moment arm from the hand force sensor made the device easier to rotate when subjected to force input on the hand force sensor. This configuration more 
closely corresponds to human movement, as the lateral/medial rotation of the arm occurs along the upper arm's axis through the elbow, and not the middle of the forearm. We chose not to place the center of mass at the elbow location, as this would prevent elbow input forces from producing rotations.

For the ICR controller, which does not have a center of mass or torque computation, we chose instead to increase the accommodation gain from $0.010(\mathrm{~m} / \mathrm{s}) / \mathrm{N}$ to $0.015(\mathrm{~m} / \mathrm{s}) / \mathrm{N}$. Our hopes here again were that this change would allow easier movement without degradation in performance. For this second experiment, we asked participants to trace straight lines in eight directions.

\section{Controller Enhancement Comparison Methods}

Lines were presented in 8 direction increments of 45 degrees: horizontal at 0 degrees, and rotated at $45,90,135,180,225,270$, and 315 degrees. The experiment was completed by 6 participants $(5$ males and 1 female, ranging in age from 25 to 40 years. The total time required to complete the experiment was approximately 40 minutes, including time for breaks between experiment conditions.

The main factors of interest in this experiment were the controller condition and the drawing direction. Each participant completed 16 trials (2 repetitions of 8 directions) for each of 6 conditions: the 3 original controllers and 3 modified controllers for the Basic, ICR, and FBA conditions.

For this experiment, we used a two-way $6 \times 8$ factorial ANOVA to predict median error by controller condition and drawing direction with participant number entered as a random variable. We examined a two-way $6 \times 8$ factorial ANOVA to predict mean completion time by controller condition and drawing direction with participant number entered as a random variable. We then used a two-way $6 \times 8$ factorial ANOVA to predict total integrated force and efficiency by controller condition and drawing direction with participant number entered as a random variable.

\section{Controller Enhancement Comparison Results and Discussion}

Controller condition did not significantly predict median error $(F[5,287]=2.52, p=.06)$ (Fig. $6(\mathrm{a})$ ). When examining the pairs of controller conditions (original and modified), there was no difference in the median error for the Basic and FBA conditions $(p>0.55)$. However, the modified ICR condition had significantly higher error than the original ICR condition by $0.039 \mathrm{~mm}(p$ $<.01)$. Drawing direction significantly predicted median error $(F[7,287]=5.54, p<.01)$. Drawing in the orthogonal directions (bottom to top, top to bottom, left to right, and left to right) had the lower median error than drawing in the non-orthogonal directions by $0.128 \mathrm{~mm}(p<.035)$.

Controller condition also did not significantly predict completion time $(F[5,287]=0.21, p$ $=.95)$ (Fig. 6(b)). When examining the pairs of controller conditions, there was no difference in the median error for any of the conditions $(p>0.070)$. Drawing direction significantly predicted completion time $(F[7,287]=13.52, p<.01)$. There were three levels of completion times. Drawing horizontally (left to right or right to left) was significantly faster than the other 6 directions at an average of $3.89 \mathrm{~s}$. Drawing vertically (top to bottom or bottom to top), drawing from bottom left to top right, and drawing from top right to bottom left was next fastest with an average completion time of $4.54 \mathrm{~s}(p<.01)$, and drawing from top left to bottom right or from bottom right to top left was the slowest, with an average completion time of $5.21 \mathrm{~s}(p<.01)$.

Controller condition significantly predicted total integrated force, which is an indicator of the effort required during each trial $(F[5,287]=29.65, p<.01)$ (Fig. 6(c)). There were three levels of total integrated force. The original Basic and FBA controllers had the lowest total integrated force at an average of 13.18 Ns. The original and modified ICR and the modified FBA controllers had the next lowest total integrated force at an average of $17.19 \mathrm{Ns}(p<.01)$. The modified Basic controller had the highest total integrated force at $20.02 \mathrm{Ns}(p<.01)$. From this examination, it can be seen that the original basic and original FBA controllers had lower total integrated force than their corresponding modified controllers. 
Drawing direction also significantly predicted total integrated force $(F[7,287]=41.08, p$ $<.01)$. There were three levels of total integrated force as a function of drawing direction. Drawing horizontally had the lowest total integrated force at an average of 12.59 Ns. Drawing from bottom left to top right (on the display screen) and drawing from top right to bottom left had the next lowest total integrated force at an average of 14.98 Ns $(p<.01)$. The remaining 4 directions had the highest total integrated force at an average of $18.86 \mathrm{Ns}(p<.01)$.

Additionally, there was a significant interaction between controller condition and drawing direction when predicting total integrated force $(F[35,287]=12.61, p<.01)$. Drawing horizontally, drawing from bottom left to top right, and drawing from top right to bottom left took significantly less total integrated force than the other four directions for the original ICR and all three modified controllers. The original Basic and FBA controllers showed no differences in total integrated force by drawing direction.

Controller condition significantly predicted efficiency $(F[5,287]=30.64, p<.01)($ Fig. $6(d))$. The original FBA and Basic controllers had lower efficiency than any of the other controller conditions $(\mathrm{p}<.01)$. Drawing direction also significantly predicted efficiency $(F[7,287]=7.77, p$ $<.01)$. Drawing horizontally, drawing from bottom left to top right, and drawing from top right to bottom left were significantly less efficient than the other four directions. There was also a significant interaction between controller condition and drawing direction when predicting efficiency $(F[35,287]=3.45, p<.01)$. Drawing horizontally, drawing from bottom left to top right, and drawing from top right to bottom left were significantly less efficient than the other four directions for the original Basic and FBA controllers and the modified ICR controller. The modified Basic and FBA controllers and the original ICR controller showed no differences in efficiency by drawing direction.

We finally examined the subjective data to choose between the pairs of controller conditions (original and modified). When asked how natural the controller condition felt, participants preferred the modified Basic condition (4.0 vs. 3.2/5.0), the original ICR (3.9 vs. 3.2/5.0), and the modified FBA (4.1 vs. 3.7/5.0).
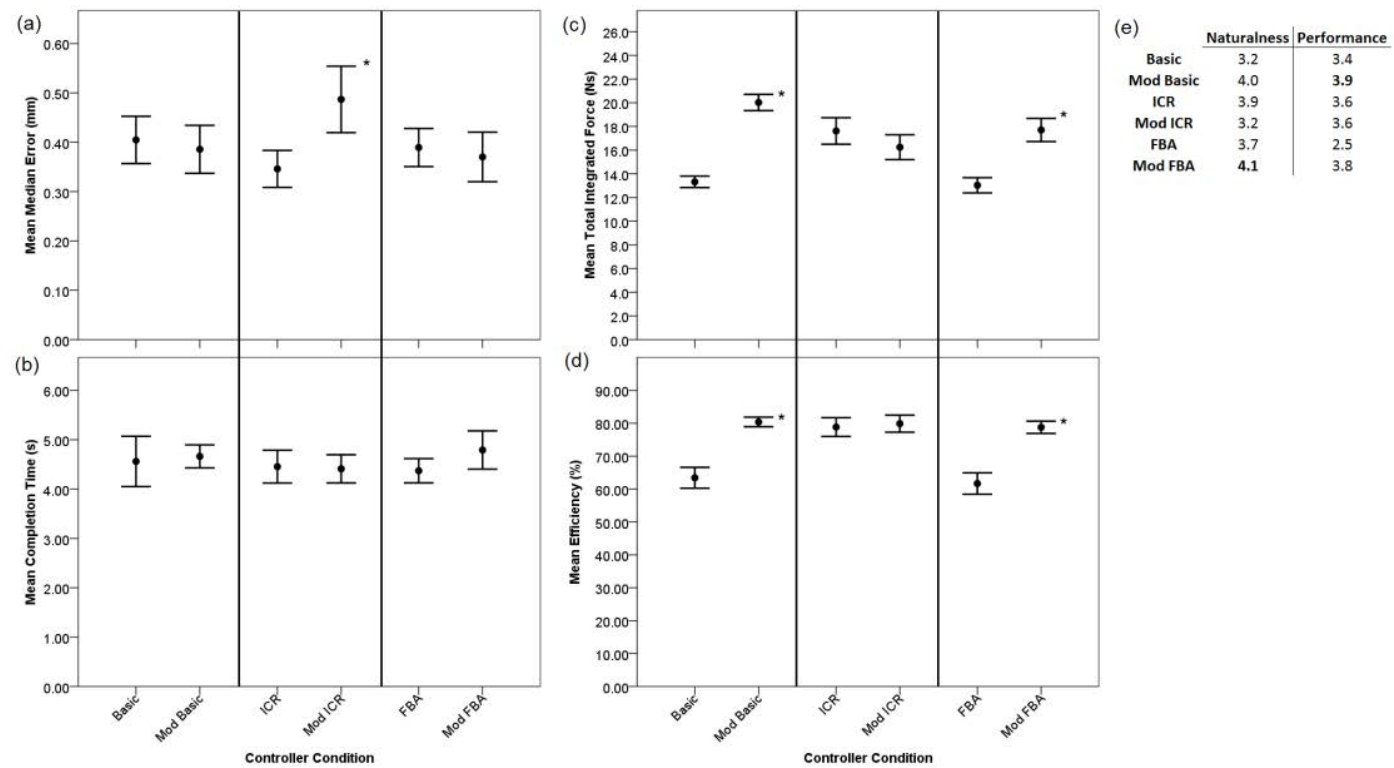

Figure 6. Experiment 2 Median Error (a), Completion Time per Distance (b), Total Integrated Force per Distance (c), Efficiency (d), and Subjective Ratings (e) by Contoller Condition. (Error bars indicate 95\% confidence intervals for the estimated mean.) 
Fehlberg et al., Enhancements to the Planar Active Handrest

When asked how well they perceived that they performed, participants felt that they did better with the modified Basic (3.9 vs. 3.4/5.0) and the modified FBA (3.8 vs. 2.5/5.0). There was no difference between the original and modified ICR. Participants subjectively preferred the modifications for the Basic and FBA controllers but preferred the original ICR controller.

Based on the efficiency and subjective findings, we decided to use the modifications to the Basic and FBA controller conditions while retaining the original configuration for the ICR controller in our final experiment.

\section{Experiment 3 - E-PAHR, PAHR, and Freehand Comparison}

We conducted our third experiment to compare the performance of the selected E-PAHR controller strategies to the non-rotating PAHR and the Freehand (unsupported) conditions. The only factor of interest in this experiment was the controller/support condition. Focusing specifically on the controller/support condition allowed us to examine the error versus completion time trade-off through a hierarchical regression analysis.

E-PAHR, PAHR, and Freehand Comparison Methods

We first desired to confirm the relevant results from Experiment 2 for the data from Experiment 3. For Experiment 3, each support condition of the experiment had participants trace 3 repetitions of small sine wave, oblate limacon, asymmetric lemniscate, and rectangle shapes, and then trace 3 repetitions of larger shapes ( 1.5 times wider) for each shape type. The experiment was completed by 20 participants ( 17 males and 3 females, ranging in age from 21 to 41 years old) of which 2 were authors of this paper. The total time required to complete the experiment was approximately 60 minutes, which included time for breaks between experiment conditions.

The main factor of interest in this experiment was the fixture condition. Each participant completed 16 trials (4 shapes $\mathrm{x} 2$ repetitions $\mathrm{x} 2$ sizes) under each of the 5 support conditions: 3 with the E-PAHR, 1 with the PAHR, and 1 Freehand. For this experiment, the smaller shapes were presented as the same size from Experiment 1, and the larger shapes were approximately 1.5 times larger in width along the displayed X-axis. Due to the PAHR's limited workspace, participants were given four repetitions of the smaller shapes and no larger shapes.

For this experiment, we used a two-way $4 \times 5$ factorial ANOVA to predict median error by shape and support condition with participant number entered as a random variable. We examined a two-way 4 x 5 factorial ANOVA to predict mean completion time per path length by shape and support condition with participant number entered as a random variable. We then used a two-way 4 x 5 factorial ANOVA to predict total integrated force and efficiency by support condition and shape with participant number entered as a random variable. Finally, we used a two-way 4 x 5 factorial ANOVA to predict the perceived level of exertion by support condition and shape with participant number entered as a random variable.

E-PAHR, PAHR, and Freehand Comparison Results and Discussion

Support condition predicted median error $(F[4,454]=49.49, p<.01)$ (Fig. 7(a)). The Freehand condition had the greatest amount of median error at $0.76 \mathrm{~mm}(p<.01)$. The PAHR condition at $0.56 \mathrm{~mm}$ had less median error than the Freehand condition and more median error than any of the E-PAHR conditions by an average of $0.06 \mathrm{~mm}(p<.01)$. Within the E-PAHR support conditions, the FBA controller had more median error than the ICR controller by $0.03 \mathrm{~mm}(p=.01)$. Shape type also predicted median error $(F[7,454]=13.71, p<.01)$. Small $(0.16 \mathrm{~m} \mathrm{x} 0.08 \mathrm{~m})$ and large $(0.24 \mathrm{~m} \times 0.08 \mathrm{~m})$ rectangles had less median error than the other shapes $(p<.01)$.

Before conducting completion time analyses, we again normalized the completion time per path length. Support Condition predicted time per path length $(F[4,454]=6.79, p<.01)$ (Fig. $7(\mathrm{~b}))$. Freehand had the lowest time per path length at $41.47 \mathrm{~s} / \mathrm{m}(p<.01)$. The PAHR condition had less time per path length than Basic and ICR conditions by an average of $2.89 \mathrm{~s} / \mathrm{m}(p<.01)$, 
Fehlberg et al., Enhancements to the Planar Active Handrest
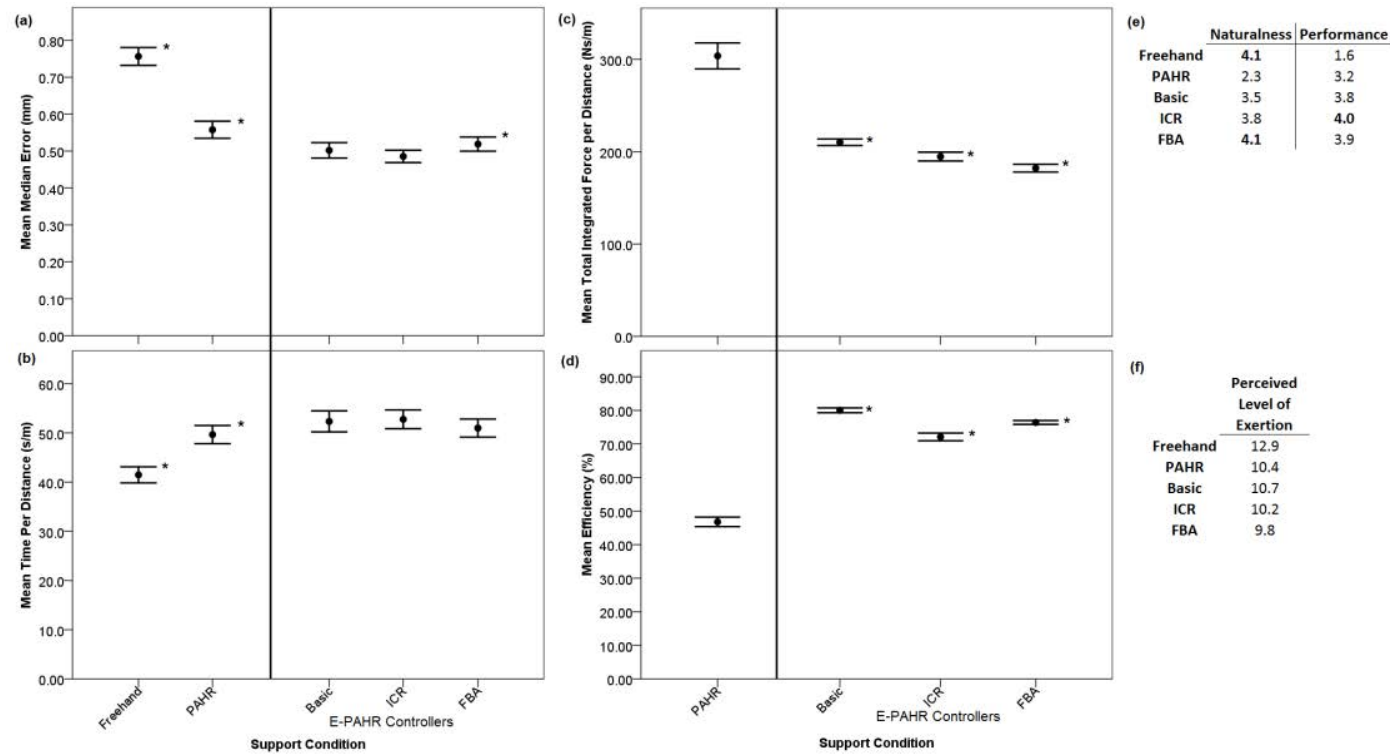

Figure 7. Experiment 3 Median Error (a), Completion Time per Distance (b), Total Integrated Force per Distance (c), Efficiency (d), Subjective Ratings (e), and Perceived Level of Exertion by Contoller Condition. (Error bars indicate $95 \%$ confidence intervals for the estimated mean.)

but the same time per path length as the FBA condition $(p=.185)$. Shape type predicted time per path length $(F[7,454]=31.70, p<.01)$. Small and large sine waves took more time per distance than the other shapes $(p<.01)$.

All three E-PAHR controller conditions had less median error but higher completion time per path length than the PAHR and Freehand. To test the hypothesis that there is a speed versus accuracy trade-off (cf. Fitts, 1954) in our task, we conducted a hierarchical multiple regression. Due to the general inverse relationship between error and task completion time as found both here and in Fehlberg et al., (2012), we first entered the completion time per distance and the inverse of completion time per distance as a set into the hierarchical regression model, followed by use of a device (either the PAHR or E-PAHR), and finally, use of the E-PAHR.

Hierarchical regression analysis indicates that the completion time per distance set accounts for a significant $27.1 \%$ of the variance in median error $(F[2,1583]=294.69, p<.01)$. Use of a device (PAHR or E-PAHR) accounts for an additional significant $9.9 \%$ of the variance in median error $(F[1,1582]=249.90, p<.01)$, leading to an average reduction in error of $0.14 \mathrm{~mm}$, which confirms the findings of Fehlberg et al. (2012) and Fehlberg et al. (2014) that use of the Active Handrest leads to an increase in user skill level. Use of the E-PAHR accounts for an additional significant but small $0.6 \%$ of the variance in median error $[(F 1,1581]=15.99, \mathrm{p}<.01)$, leading to an average reduction in error of $0.04 \mathrm{~mm}$. There was no discernable difference in skill level between the three E-PAHR controllers.

Support condition significantly predicted total integrated force $(F[3,681]=18.41, p<.01)$ (Fig. 7(c)). The E-PAHR's FBA controller had the lowest total integrated force per distance at $182.18 \mathrm{Ns} / \mathrm{m}$. The ICR controller had the next lowest total integrated force per distance at 194.69 $\mathrm{Ns} / \mathrm{m}(p<.01)$, followed by the Basic controller at $210.25 \mathrm{Ns} / \mathrm{m}(p<.01)$. The PAHR had the highest total highest total integrated force per distance at $303.76 \mathrm{Ns} / \mathrm{m}(p<.01)$. From this examination, it can be seen that all of the E-PAHR controllers had significantly lower total integrated force per distance than the PAHR, hence the user had lower exertion when using the EPAHR than PAHR. 
Fehlberg et al., Enhancements to the Planar Active Handrest

Shape type also significantly predicted total integrated force $(F[7,681]=46.23, p<.01)$. Small (0.1 m wavelength) and large (0.15 m wavelength) sine waves, each of amplitude $0.05 \mathrm{~m}$, had higher total integrated force per distance than the other shapes $(p<.01)$. There was a significant interaction between support condition and shape type $(F[17,681]=4.23, p<.01)$. This interaction was caused by sine waves requiring more total integrated force per distance under the 3 E-PAHR conditions than with the PAHR.

Support condition significantly predicted efficiency $(F[3,681]=92.40, p<.01)($ Fig. $7(d))$. The PAHR had lower efficiency than any of the E-PAHR controller conditions at $46.80 \%$ ( $p$ $<.01)$. The ICR controller had the next lowest efficiency at $72.09 \%(p<.01)$ followed by the Basic controller at $76.40 \%(p<.01)$. The FBA controller had the highest efficiency at $80.02 \%$.

Shape type also significantly predicted efficiency $(F[7,681]=12.66, p<.01)$. Drawing the smaller shapes had significantly lower efficiency than the larger shapes. This was caused by the interaction between support condition and shape type when predicting efficiency $(F[17,681]=$ $9.66, p<.01)$. Small shapes traced using the PAHR had significantly lower efficiency than when traced with any of the E-PAHR conditions.

Lastly, we examined the subjective data ratings for naturalness, performance, and perceived level of exertion. When asked how natural the controller condition felt, participants equally preferred the FBA and Freehand conditions (4.1/5.0). The PAHR condition was perceived to be the least natural condition (2.3/5.0). When asked how well they perceived that they performed, participants felt that they did slightly better with the E-PAHR's ICR controller (4.0/5.0) compared to the FBA and Basic controllers (3.9 and 3.8/4.0).

Participants also felt they performed worse with the PAHR condition (3.2/5.0) and the worst overall with the Freehand condition (1.6/5.0). These perceptions were supported by the findings for median error and from the hierarchical regression as discussed earlier in this section.

When asked about their perceived level of exertion, participants found Freehand to have the highest level of perceived exertion (12.9/20). Participants perceived the E-PAHR's FBA controller to require the lowest level of exertion (9.8/20). The perception that the FBA controller had the lowest exertion was supported by the measured total integrated force per distance. Surprisingly, participants found the PAHR, Basic, and ICR conditions to have about the same level of perceived exertion $(10.4,10.7$, and 10.2 out of 20), while the measured total integrated force per distance was actually much higher for the PAHR condition.

Based on the lower perceived and actual (low total integrated force) levels of exertion, participant ratings for Naturalness, relatively high Efficiency, and high skill level, we determined that the FBA controller was the best strategy for control of the Enhanced Planar Active Handrest. This controller has the additional benefit of being the most flexible by allowing specific virtual masses and damping (inverse of admittance gain) for both translations and rotations.

\section{Conclusions}

In this work, we endeavored to improve upon the performance of the Active Handrest, a planar device that assists a user in performing precision manipulation tasks over an extended workspace. The results discovered expand on the previous studies of the Active Handrest (Fehlberg et al., 2012; Fehlberg et al., 2014), which showed a skill level improvement while using the device for large workspace precision tracing tasks. To improve the performance of the device, we explored the effectiveness of a rotational degree-of-freedom to the Active Handrest. Our first experiment helped us to identify the most effective controller strategy for the E-PAHR.

After selecting the Basic, ICR, and FBA as the most effective controller strategies for the EPAHR, we then conducted a second experiment to attempt to improve these three strategies. We decided to use the modifications to the Basic and FBA controllers but retain the original ICR controller.

Our third and final experiment compared these three E-PAHR controller strategies to the 
Fehlberg et al., Enhancements to the Planar Active Handrest

original PAHR and Freehand support conditions. Through our analysis, we determined that after accounting for the effects of completion time, use of the E-PAHR led to significantly less median error than Freehand and slightly less median error than use of the PAHR. Subjective analysis also showed user preference for the E-PAHR over the PAHR. Additional analysis of user force input showed a large reduction in the total integrated force required to complete a task and an almost doubled efficiency for the E-PAHR over the PAHR. These findings, along with the E-PAHR's increased workspace, underscore the potential benefit for the Active Handrest's use in conducting large workspace precision tasks.

Through our experimentation, we demonstrated that the E-PAHR is a useful device for assisting users in performing precision tasks over a large workspace. We have confirmed the finding that the E-PAHR improves user skill level, and we established that the E-PAHR reduces user effort when compared to our previous device prototype. Based on these results, we anticipate that the Enhanced Planar Active Handrest will be useful in many practical applications, such as medical tasks, artistry, machining, upper limb rehabilitation, or other tasks requiring dexterous manipulation of tools while preforming precision tasks over a large workspace. In the future, we intend to develop a Spatial Active Handrest that will allow for support in the vertical direction and increased precision as a user performs tasks in a three-dimensional large workspace.

\section{Acknowledgements}

The authors thank Andrew Doxon for his support in developing and implementing the device controller. This work was supported, in part, by the National Science Foundation under awards IIS-0746914, IIS-0904456, and DGE-0654414.

\section{References}

Beer, R. F., Ellis, M. D., Holubar, B. G., \& Dewald, J. P. A. (2007). Impact of gravity loading on post-stroke reaching and its relationship to weakness. Muscle \& Nerve, 36(2), 242-250. doi: $10.1002 /$ mus.20817

Beer, R. F., Naujokas, C., Bachrach, B., \& Mayhew, D. (2008). Development and evaluation of a gravity compensated training environment for robotic rehabilitation of post-stroke reaching. In Proceedings of the 2nd IEEE RAS \& EMBS International Conference on Biomedical Robotics and Biomechatronics (pp. 205-210). doi:10.1109/BIOROB.2008.4762863

Ben-Tov, M., Levy-Tzedek, S., \& Karniel, A. (2012). The effects of rhythmicity and amplitude on transfer of motor learning. PloS ONE, 7(10). doi:10.1371/journal.pone.0046983

Borg, G. (1982). Psychophysical bases of perceived exertion. Medical Science Sports Exercise, 14(5), 377-381. doi:10.1249/00005768-198205000-00012

Borg G. (1990). Psychophysical scaling with applications in physical work and the perception of exertion. Scandinavian Journal of Work, Environment \& Health, 55-58. doi:10.5271/sjweh. 1815

Chyatte, S. B., Long II, C., \& Vignos Jr., P. J. (1965). The balanced forearm orthosis in muscular dystrophy. Archives of Physical Medicine and Rehabilitation, 46, 633-636.

Colgate, J., Wannasuphoprasit, W., \& Peshkin, M. (1996). Cobots: Robots for collaboration with human operators. In Proceedings of the International Mechanical Engineering Congress and Exhibition (Vol. DSC-58, pp. 433-440).

Colombo, R., Pisano, F., Micera, S., Mazzone, A., Delconte, C., Carrozza, M. C., Dario, P., \& Minuco, G. (2005). Robotic techniques for upper limb evaluation and rehabilitation of 
Fehlberg et al., Enhancements to the Planar Active Handrest

stroke patients. IEEE Transactions on Neural Systems and Rehabilitation Engineering, 13(3), 311-324. doi:10.1109/TNSRE.2005.848352

Erdelyi A., Sihvonen, T., Helin, P., \& Hanninen O. (1998). Shoulder strain in keyboard workers and its alleviation by arm supports. International Archives of Occupational and Environmental Health, 60, 119-124. doi:10.1007/BF00381492

Equipois (2015). ZeroG. Retrieved from http://www.equipoisinc.com

Fehlberg, M., Gleeson, B., \& Provancher, W. (2012). Active Handrest: A large workspace tool for precision manipulation. The International Journal of Robotics Research, 31(3), 289-301. doi:10.1177/0278364911432895

Fehlberg, M., Nisky, I., Doxon, A., \& Provancher, W. (2014). Improved active handrest performance through use of adaptive admittance and virtual fixtures. IEEE Transactions on Human-Machine Systems, 44(4), 484-498. doi:10.1109/THMS.2014.2321531

Fitts, P. (1954). The information capacity of the human motor system in controlling the amplitude of movement. Journal of Experimental Psychology, 47(6), 381-391. doi: $10.1037 / \mathrm{h} 0055392$

Goncalves, L., Di Bernardo, E., Ursella, E., \& Perona, P. (1995). Monocular tracking of the human arm in 3D. In Proceedings of the 5th International Conference on Computer Vision, (pp. 764-770). doi:10.1109/ICCV.1995.466861

Hogan, N. (1985). Impedance control: An approach to manipulation: Part III applications. Journal of Dynamic Systems, Measurement, and Control, 107(2), 17. doi:10.1115/1.3140701

Hogan, N., Krebs, H. I., Sharon, A., \& Charnnarong, J. (1995). Interactive robotic therapist. US Patent 5466213A, Massachusetts Institute of Technology.

Hogan, N. \& Sternad, D. (2007). On rhythmic and discrete movements: Reflections, definitions and implications for motor control. Experimental Brain Research, 181(1), 13-30. doi:10.1007/s00221-007-0899-y

Ito, S. and Yokokohji, Y. (2009). Maneuverability of master control devices considering the musculo-skeletal model of an operator. In Proceedings of the 3rd Joint EuroHaptics Conference and Symposium on Haptic Interfaces for Virtual Environment and Teleoperator Systems (World Haptics) (pp. 57-62). doi:10.1109/WHC.2009.4810801

Krakauer, J. W. \& Mazzoni, P. (2011). Human sensorimotor learning: Adaptation, skill, and beyond. Current Opinion in Neurobiology, 21(4), 636-644. doi:10.1016/j.conb.2011.06.012

Kwakkel, R., Wagenaar, C., Koelman, T. W., Lankhorst, G. J., \& Koetsier, J. C. (1997). Effects of intensity of rehabilitation after stroke: A research synthesis. Stroke, 28(8), 1550-1556. doi:10.1161/01.STR.28.8.1550

Langhammer, B., Lindmark, B., \& Stanghelle, J. K. (July 2007). Stroke patients and long-term training: Is it worthwhile? A randomized comparison of two different training strategies after rehabilitation. Clinical Rehabilitation, 21(6), 495-510. doi:10.1177/0269215507075207

Levy-Tzedek, S., Ben-Tov, M., \& Karniel, A. (2011). Early switching between movement types: Indication of predictive control? Brain Research Bulletin, 85(5), 283-288. doi:10.1016/j.brainresbull.2010.11.010

Likert, R. (1932). A technique for the measurement of attitudes. Archives of psychology 22(140), $1-55$.

Loureiro, R., Amirabdollahian, F., Topping, M., Driessen, B., \& Harwin, W. (July 2003). Upper limb robot mediated stroke therapy-GENTLE/s approach. Autonomous Robots, 15(1), 35-51. doi:10.1023/A:1024436732030 
Fehlberg et al., Enhancements to the Planar Active Handrest

Lum, P. S., \& Burgar, C. G. (2002). Robot-assisted movement training compared with conventional therapy techniques for the rehabilitation of upper-limb motor function after stroke. Archives of Physical Medicine and Rehabilitation, 83(7), 952-959. doi:10.1053/apmr.2001.33101

Mathewson, B. B. \& Newman, W. S. (1995). Integration of force strategies and natural admittance control. In Proceedings of the IEEE Robotics and Automation Conference (Vol. 3, pp V6). doi:10.1109/ROBOT.1995.526010

Matsuo, S. \& Iwaki, S. (1992). Compliance design using linear programming. In Proceedings of the IEEE Robotics and Automation Conference, (Vol. 2, pp. 1238-1234). doi:10.1109/ROBOT.1992.220079

Moeslund T. B. \& Granum, E. (2000). Multiple cues used in model-based human motion capture. In Proceedings of the 4th IEEE International Conference on Automatic Face and Gesture Recognition (pp. 362-367). doi:10.1109/AFGR.2000.840660

Moubarak, S., Pham, M., Moreau, R., \& Redarce, T. (2010). Gravity compensation of an upper extremity exoskeleton. In Proceedings of the IEEE Annual International Conference on Engineering in Medicine and Biology Society (pp. 4489-4493). doi:10.1109/IEMBS.2010.5626036

Nambi, M., Provancher, W., \& Abbott J. (2011). On the ability of humans to apply controlled forces to admittance-type devices. Advanced Robotics, 25, 629-650. doi:10.1163/016918611X558234

Nef, T., Guidali, M., \& Riener, R. (2009). ARMin III - Arm therapy exoskeleton with an ergonomic shoulder actuation. Applied Bionics and Biomechanics, 6(2), 127-142. doi:10.1080/11762320902840179

Plamondon, R. \& Alimi, A. M. (1997). Speed/accuracy trade-offs in target-directed movements. Behavioral and Brain Sciences, 20(2), 279-303. doi:10.1017/S0140525X97001441

Riener, R., Nef, T., \& Colombo, G. (2005). Robot-aided neurorehabilitation of the upper extremities. Medical and Biological Engineering and Computing, 43(1), 2-10. doi:10.1007/BF02345116

Reis, J., Schambra, H. M., Cohen, L. G., Buch, E. R., Fritsch, B., Zarahn, E., Celnik, P. A., \& Krakauer, J. W. (2009). Noninvasive cortical stimulation enhances motor skill acquisition over multiple days through an effect on consolidation. In Proceedings of the National Academy of Sciences (Vol. 106, No. 5), pp. 1590-1595). doi:10.1073/pnas.0805413106

Schaal, S., Sternad, D., Osu, R., \& Kawato, M. (2004). Rhythmic arm movement is not discrete. Nature Neuroscience, 7(10), 1136-1143. doi:10.1038/nn1322

Shmuelof, L., Krakauer, J. W., \& Mazzoni, P. (2012). How is a motor skill learned? Change and invariance at the levels of task success and trajectory control. Journal of Neurophysiology, 108(2), 578-594. doi:10.1152/jn.00856.2011

Taylor, R., Jensen, P., Whitcomb, L., Barnes, A., Kumar, R., Stoianovici, D., Gupta, P., Wang, Z., Dejuan, E., \& Kavoussi, L. (1999). A steady-hand robotic system for microsurgical augmentation. The International Journal of Robotics Research, 18(12), 1201-1210. doi:10.1177/02783649922067807

Ueberle, M., Mock, N., \& Buss, M. (2004). VISHARD10, a novel hyper-redundant haptic interface. In Proceedings of the 12th International Symposium on Haptic Interfaces for Virtual Environment and Teleoperator Systems (pp. 58-65). doi:10.1109/HAPTIC.2004.1287178

Van der Linde, R. Q., Lammertse, P., Frederiksen, E., \& Ruiter, B. (2002). The HapticMaster, a new high-performance haptic interface. In Proceedings of EuroHaptics (pp. 1-5). 
Whitney, Daniel E. (1977). Force feedback of manipulator fine motions. ASME Journal of Dynamic Systems, Measurement, and Control, 99, 91-97. doi:10.1115/1.3427095

Wise, S. P., \& Shadmehr, R. (2002). Motor control. Encyclopedia of the human brain, 3, 137-157. doi:10.1016/B0-12-227210-2/00216-8

Woodworth, R. S. (1899). Accuracy of voluntary movement. The Psychological Review: Monograph Supplements, 3(3), i-114. doi:10.1037/h0092992

Yasuda, Y. L., Bowman, K., \& Hsu, J. (1986). Mobile arm supports: Criteria for successful use in muscle disease patients. Archives of Physical Medicine and Rehabilitation, 67, 253-256.

Authors' names and contact information: M. A. Fehlberg, Department of Mechanical Engineering, University of Utah, Salt Lake City, USA. Email: m.fehlberg@utah.edu; H. N. Sani, Department of Mechanical Engineering, University of Utah, Salt Lake City, USA. Email: hamidsani8@gmail.com; W. R. Provancher, Department of Mechanical Engineering, University of Utah, Salt Lake City, USA. Email: wil@ mech.utah.edu. 